Mots. Les langages du politique

$73 \mid 2003$

Les discours de la guerre

\title{
La « révolution militaire ». Quelques éléments historiographiques
}

"Military revolution". A few historiographical elements

La « revolución militar». Algunos elementos historiográficos

\section{Laurent Henninger}

\section{OpenEdition}

Journals

Édition électronique

URL : https://journals.openedition.org/mots/16312

DOI : $10.4000 /$ mots. 16312

ISSN : 1960-6001

Éditeur

ENS Éditions

Édition imprimée

Date de publication : 1 novembre 2003

Pagination : 87-94

ISBN : 2-84788-043-7

ISSN : 0243-6450

\section{Référence électronique}

Laurent Henninger, «La « révolution militaire ». Quelques éléments historiographiques », Mots. Les

langages du politique [En ligne], 73 | 2003, mis en ligne le 09 octobre 2008, consulté le 22 avril 2022.

URL : http://journals.openedition.org/mots/16312 ; DOI : https://doi.org/10.4000/mots.16312

\section{(C) ENS Éditions}




\section{La « révolution militaire ». Quelques éléments historiographiques}

Le concept de «révolution militaire» est, depuis environ quinze ans, l'objet d'une controverse au sein de la communauté des historiens de l'époque moderne. Parallèlement, chez les spécialistes des questions militaires contemporaines et prospectives, un débat sur la « révolution dans les affaires militaires » qui serait actuellement perceptible dans l'évolution des armées des grandes puissances industrialisées se déroule depuis le début des années 1990. Précisons toutefois que, surtout dans le deuxième cas, ces débats se déroulent dans les pays anglo-saxons, États-Unis en tête.

S'il semble que l'un des premiers emplois de cette locution ${ }^{2}$ doive être relevé chez Michelet (dans son récit de la bataille de Rocroi), la controverse actuelle prend sa source dans le texte d'une conférence prononcée en 1955 à l'université de Belfast par le professeur Michael Roberts, sous le titre : «The Military Revolution, 1560-1660 ». Le thème restera de longues années en friche, jusqu'à ce que, en 1976, un jeune universitaire britannique, Geoffrey Parker, publie un article critiquant M. Roberts : « The «Military Revolution, 1560-1660»-A Myth ? ». En 1988, G. Parker publie ses travaux dans un ouvrage plus conséquent, qui sera ensuite traduit en français (pour l'ensemble des références, voir la bibliographie), chose suffisamment rare dans ce domaine historique si particulier qu'est l'histoire dite "militaire» pour être relevée. Il nous semble assez clair que, pour M. Roberts comme G. Parker, la révolution militaire n'a été, en quelque sorte, qu'une transposition dans un autre domaine du concept de « révolution industrielle ». Il est d'ailleurs intéressant de constater que nous avons là une similitude de plus entre l'histoire économique et l'histoire militaire, deux disciplines-sœurs par bien des

1. Centre d'études d'histoire de la Défense (CEHD) - Château de Vincennes - BP 153 00841 ARMÉES - Ihenninge1@noos.fr

2. Pour plus de précisions sur le mot révolution lui-même, on consultera avec profit l'entrée consacrée à ce mot dans le Dictionnaire historique de la langue française, d'A. Rey, ainsi que, du même auteur, 1989, "Révolution », histoire d'un mot, Paris, Gallimard. 
aspects, mais qui ne le savent pas encore. Peu après, un autre spécialiste britannique des questions militaires à l'époque moderne, Jeremy Black, de l'université d'Exeter, publie un petit essai s'inscrivant contre la thèse de G. Parker. Depuis lors, le débat s'est poursuivi et cette question a fait l'objet de nombreuses études tout au long des années 1990.

Or, il apparait que cette controverse n'a pas encore été véritablement tranchée. Chacun est plus ou moins resté campé sur ses positions et continue ses travaux dans sa direction originelle, tout en entretenant des rapports courtois et parfaitement scientifiques avec ses « adversaires ». En bref, la question de la révolution militaire des temps modernes reste toujours en suspens. Un pas important en direction d'une synthèse a toutefois été franchi en 1995 avec la publication d'un ouvrage collectif sous la direction de Clifford J. Rogers, jeune professeur d'histoire à l'académie militaire américaine de West Point et médiéviste spécialisé dans les aspects militaires de la guerre de Cent Ans : The Military Revolution Debate - Readings on the Military Transformation of Early Modern Europe. Ce volume contient en effet l'essentiel des textes fondateurs de ce débat (M. Roberts, G. Parker, J. Black, C. J. Rogers, J. Lynn, D. A. Parrott, J. F. Guilmartin), enrichis d'autres contributions abordant des points particuliers et d'une excellente introduction en forme de synthèse conceptuelle, due à Rogers lui-même. Si le débat n'est toujours pas dominé par une thèse faisant autorité, il n'en reste pas moins que l'expression est désormais entrée dans le vocabulaire courant des historiens modernistes et semble devoir y rester longtemps, ce qui constitue une sorte de validation de facto de ce concept, même s'il est parfois utilisé par ses adversaires. Mais il semble aujourd'hui quelque peu «en sommeil», car les polémiques sur cette question sont aujourd'hui "gelées » au point atteint il y a maintenant presque dix ans sans qu'aucune thèse ne l'ait véritablement emporté. Aujourd'hui, tous les acteurs de ce débat chez les historiens s'accordent au moins à reconnaitre que les façons et les moyens de faire la guerre des Européens ont connu des changements majeurs dans une période s'étendant grossièrement de la fin du Moyen Âge au $18^{\mathrm{e}}$ siècle. Tous reconnaissent en outre que ces changements ont finalement abouti à la domination du monde par l'Occident.

Sur quels points ont porté ces controverses ? D'abord sur des questions de chronologie et de datation. Cette série d'évènements s'est-elle produite dans la première moitié du $16^{\mathrm{e}}$ siècle (guerres d'Italie) ? Dans la seconde (guerre hispano-hollandaise)? Dans la première moitié du $17^{\mathrm{e}}$ siècle (guerre de Trente Ans) ? Ou bien dès le $15^{\mathrm{e}}$ siècle (guerre de Cent Ans, renouveau de l'infanterie lourde en Europe occidentale et centrale - milices communales italiennes et flamandes, archers anglais, contingents suisses, armées hussites 
de Bohème) ? J. Black, quant à lui, penchant plutôt en faveur du $18^{\mathrm{e}}$ siècle. $\mathrm{Au}$ final, il apparaitrait que toutes ces périodes peuvent être retenues et mises bout à bout pour former une vaste période de bouleversements se succédant pratiquement sans interruption pour aboutir à une relative stabilisation à la fin du règne de Louis XIV.

Cela pose alors une autre question, sans doute épistologiquement bien plus intéressante et complexe. Car peut-on encore parler de « révolution » dès lors qu'un processus s'étale sur plus de trois siècles ? Ne devrait-on pas plutôt parler d' " évolution »? Il nous semble possible de répondre par la négative à cette dernière question, et ce, pour plusieurs raisons. D'une part, on parle bien de "révolution néolithique», alors que celle-ci s'étale sur plusieurs millénaires ; dans ce cas, l'emploi du vocable révolution peut se justifier par l'insistance qu'il contient sur la notion d'accélération d'un processus. Ensuite, cette série de changements militaires qui se succèdent à la fin du Moyen Âge et à l'époque moderne apparait systématiquement comme étroitement liée aux bouleversements connus par le monde occidental durant la même période (sociaux, politiques, économiques, scientifiques, culturels et même géographiques, avec les Grandes Découvertes et le début de la conquête du monde par l'Occident). En ce sens, elle n'est que l'une des facettes de la naissance de la modernité et prend donc ainsi tout son sens en tant que tournant macrohistorique majeur. L'histoire militaire n'est, en cela, pas différente de l'histoire "générale ", et les changements révolutionnaires enregistrés à l'époque moderne apparaissent comme le résultat combiné de nouvelles technologies, d'innovations tactiques et organisationnelles, et de nouvelles façons de penser interagissant avec le Zeitgeist. Reste la question des mots : révolution, évolution, changement, transformation, rupture ? Cette problématique nous semble avoir été tranchée, de façon quasi définitive cette fois, par C. J. Rogers. Dans le texte précité, cet auteur propose d'appliquer à cette problématique les critères employés en biologie évolutionniste par les néodarwiniens, et plus précisément ce qu'ils dénomment la théorie de l'équilibre ponctué. Ces derniers - en particulier Stephen Jay Gould et Niles Eldredge - ont en effet modifié les théories de Charles Darwin en complétant l'idée - soutenue originellement par ce dernier - selon laquelle l'évolution des espèces est un processus fait d'une suite de modifications mineures se succédant de façon continue, gradualiste et quasi régulière. Selon les néodarwiniens, en effet, l'évolution des espèces est bien plus chaotique : durant de très longues périodes, les changements mineurs s'accumulent puis, lorsqu'une "masse critique » est atteinte et/ou que des circonstances extérieures particulières surviennent, des bouleversements majeurs se produisent, cette fois sur des périodes relativement courtes. Ajoutons que les théories de 
S. J. Gould et N. Eldredge se combinent avec la théorie mathématique des catastrophes. Le terme qui conviendrait alors le mieux au processus historique faisant l'objet de cet article serait ainsi celui de «mutation» militaire.

Enfin, de nombreux écrits produits durant la décennie passée ont utilisé le concept de révolution militaire pour l'appliquer à d'autres moments historiques que l'époque moderne : apparition de l'infanterie moderne à la fin de l'âge de bronze, "révolution hoplitique " grecque, révolution de la féodalité et de la chevalerie (durant le Haut Moyen Âge), etc.

Les historiens militaires contemporanéistes, quant à eux, ne se sont approprié ce concept que depuis bien plus récemment et ont étudié les « révolutions militaires » liées à l'industrialisation et à la mécanisation de la guerre et des opérations (encore une fois, nous parlons là essentiellement d'historiens anglo-saxons). On voit ainsi fleurir depuis quelques années les publications consacrées aux bouleversements provoqués par la guerre de Sécession américaine (mais certains d'entre eux préfèrent remonter à l'apparition des guerres «de masse », avec la Révolution française et le Premier Empire), par la Première Guerre mondiale, ou encore à la naissance des tactiques mécanisées (emploi des blindés et de l'aviation, principalement) dans les armées de l'entre-deux-guerres. Il convient aussi de signaler que, dès les années 1960 et 1970, les théoriciens de la stratégie et des relations internationales parlaient - à juste titre, selon nous - de la « révolution de l'arme nucléaire $»$.

Or, ces travaux sur les innovations techniques et tactiques de l'entre-deuxguerres sont apparus récemment, à l'appui des études menées, par ailleurs, dans les milieux de défense américains - tant militaires que civils - sur la « révolution dans les affaires militaires » que ces mêmes milieux croient discerner et, dans le même temps, tentent d'impulser dans le gigantesque et surpuissant appareil militaire de leur pays. Comprenons donc bien qu'il ne s'agit plus là d'un débat relevant de la discipline historique, mais bien des questions politiques-stratégiques contemporaines et prospectives (même si quelques tentatives, plus ou moins maladroites, de relier ces deux problématiques et ces deux concepts ont été faites). Selon eux, cette "RMA» (Revolution in Military Affairs) serait principalement le produit de la généralisation des armes guidées à haute précision, des systèmes d'informatisation du champ de bataille ( $\mathrm{C}^{4} \mathrm{I}-$ Command, Control, Communication, Computers, Intelligence) et même de la «cyberwar » qui se mènera à l'avenir directement "à l'intérieur » - et pour le contrôle - des réseaux informatiques. L'un des principaux publicistes de ce concept, Michael Mazarr, pouvait ainsi, en 1993, définir ce qu'était selon lui une RMA : «Une avancée fondamentale en technologie ou en doctrine ou en organisation qui rend obsolète la méthode en 
vigueur de conduite de la guerre ». De son côté, un autre protagoniste de ce débat, Andrew Marshall, haut fonctionnaire au Pentagone, considérait que

le terme de «révolution » [n'était] pas destiné à insister sur le fait que le changement sera rapide - ainsi les révolutions du passé [s'étaient] étalées sur des périodes de plusieurs décennies - mais seulement à signifier que ce changement sera profond, que les nouvelles méthodes de guerre seront plus puissantes que les anciennes. Les innovations dans le domaine technologique rendent possible une révolution militaire, mais la révolution elle-même ne se produit que dès lors que de nouveaux concepts d'opérations sont développés et, bien souvent, que de nouvelles organisations militaires sont créées. La réalisation de ces changements organisationnels et doctrinaux [étant] un long processus ${ }^{3}$.

Il convient cependant de rester extrêmement prudent sur ce dernier débat, lequel a le plus souvent servi aux autorités américaines, tant civiles que militaires là encore, depuis les années 1990, à éviter un véritable débat politique sur les changements stratégiques rendus nécessaires par la disparition de l'ennemi principal de quarante ans de guerre froide. Ajoutons à cela que la plus grande confusion théorique et de graves lacunes conceptuelles sont ici monnaie courante. Il n'en reste pas moins que l'étude de ce débat est indispensable à la compréhension de la stratégie contemporaine et a fortiori future des États-Unis. Ce qui a pour corollaire de rendre nécessaire la claire compréhension qu'on n'a pas affaire là à une simple mode intellectuelle et stratégique liée à une période donnée, comme on a pu en connaitre plusieurs depuis quelques siècles, même si ce débat conserve de fortes connotations idéologiques, si ce n'est parfois politiciennes. À ce sujet, nous ne pouvons qu'inciter le lecteur à lire l'article du sociologue Alain Joxe, paru dans le Dictionnaire de stratégie dirigé par J. Klein et T. de Montbrial. Définissant «le concept de révolution dans les affaires militaires [associant] les mutations techno-scientifiques et militaires au bouleversement politique qui modifie les forces morales du soldat, et donc les rapports de force face à la menace de mort » (p. 448), A. Joxe a produit là ce qui reste sans doute la meilleure analyse critique du discours américain sur la RMA.

$\mathrm{Au}$ demeurant, les stratégistes américains ont repris ce concept, originellement baptisé « révolution technico-militaire », chez les théoriciens militaires soviétiques, lesquels, dès la seconde moitié des années 1920, ont sans doute constitué l'une des plus fascinantes écoles de pensée militaire de tout le $20^{\mathrm{e}}$ siècle, ce que l'armée américaine a reconnu puisqu'elle a adopté, dans les années 1980, un grand nombre de concepts issus des travaux de cette

3. A. Joxe, « Révolution dans les affaires militaires (concept américain de) », dans J. Klein et T. de Montbrial, 2000, p. 448-455. 
école. Dès l'entre-deux-guerres, donc, les militaires soviétiques avaient élaboré ce concept de «MTR» (Military-Technical Revolution) pour caractériser les changements intervenus avec l'industrialisation de la guerre, processus dont ils situaient le démarrage avec la guerre de Sécession. Au début des années 1930, ils allèrent plus loin que quiconque - et en particulier bien plus loin que les militaires allemands! - dans la conceptualisation de la guerre mécanisée moderne, mais aussi dans celle du niveau de conception et de direction de l'action militaire qu'était ce qu'ils avaient dénommé l'«art opératif », niveau intermédiaire entre le tactique et le stratégique. Plus tard, dans les années 1960 et 1970, ils conceptualisèrent la MTR découlant de l'apparition des armes nucléaires, et, dans les années 1980, celle découlant de l'apparition des armes de précision et de l'informatisation du champ de bataille. Le débat américain des années 1990 n'a fait, en quelque sorte, que reprendre cette question là où l'effondrement de l'URSS l'avait laissée.

Il s'agirait aujourd'hui de hiérarchiser ces "révolutions militaires », ne serait-ce que pour ne pas donner raison aux adversaires du concept, lesquels voient à juste titre dans cette véritable inflation une preuve a contrario de sa non-validité. Or, pour hiérarchiser ces révolutions, l'outil historique à lui seul est largement insuffisant, pour ne pas dire parfois impuissant. Il convient alors de faire appel à d'autres disciplines, à d'autres outils. Historiques, tout d'abord, comme l'histoire politique, l'histoire des représentations ou celle des techniques; mais aussi à des disciplines comme la sociologie ou la science politique, l'épistémologie historique, l'économie politique, la cybernétique et la science des systèmes, et enfin la sociologie, l'anthropologie et la philosophie des techniques, trois disciplines qui nous paraissent parmi les plus prometteuses en tant qu'outils « auxiliaires » destinés à dépasser le blocage dans lequel la question de la révolution militaire se trouve actuellement.

Si nous insistons sur ce dernier point, c'est que la tentation du déterminisme et du réductionnisme techniques est peut-être encore plus présente lorsqu'on aborde les questions militaires qu'avec les autres domaines historiques. Sur ce point, l'économiste Jacques Sapir, dans son article consacré au concept de révolution militaire chez les théoriciens soviétiques, paru dans le Dictionnaire de stratégie, met bien en garde contre ce danger. Évoquant les " évolutions historiques de l'art militaire conçu comme la résultante des dimensions matérielles (techniques, scientifiques, économiques), sociales et organisationnelles qui concourent à la manière dont un pays donné conduit des opérations militaires », il écrit :

Si l'innovation prend un aspect révolutionnaire, c'est à travers sa diffusion massive et sa capacité à déstabiliser les anciennes relations entre l'individu, le monde matériel et la société, et à en faire émerger de nouvelles. [...] Une révolution dans les 
affaires militaires se caractérise, du point de vue militaire, par la nécessité de changements radicaux dans la structure et la posture des forces, dans la nature du système économique et social assurant le soubassement de ces forces armées, enfin dans le rapport entre offensive et défensive, et la nature de la corrélation générale des forces. La révolution dans les affaires militaires se situe donc fondamentalement à l'intersection entre l'innovation technique, l'innovation sociale et l'innovation organisationnelle. Elle ne traduit nulle application mécanique du domaine technique sur les autres car l'innovation technique est naturellement une résultante, à la fois, des progrès économiques et scientifiques, et des transformations qui affectent les sociétés et les organisations (p. 456).

En bonne logique, Sapir en appelle donc plus loin à «penser la guerre comme un fait social total ».

On aura donc compris qu'il s'agit là, potentiellement, de l'un des chantiers historiques les plus passionnants qui soient, mais aussi un de ceux qui permettront peut-être de renouveler de façon radicale non seulement l'épistémologie de l'histoire militaire, mais également l'épistémologie de l'histoire en général, grâce à d'importantes avancées conceptuelles dans la question de l'analyse et de la définition du "changement» dans les sociétés humaines avancées que l'on est légitimement en droit d'attendre de tels travaux, puisqu'il pose de façon directe la question des forces agissantes en histoire : individus, techniques, forces sociales, politiques, économiques, mentales, etc. Enfin, l'intérêt principal de ce débat réside peut-être dans le fait suivant: pour la première fois, des historiens spécialisés dans les questions militaires tentent de penser leur objet d'étude non seulement en termes dynamiques et dialectiques, en mettant au jour les liens entre celui-ci et les autres domaines de l'activité humaine (social, économique, politique - notamment les liens entre les transformations militaires à l'époque moderne et la naissance de l'État moderne -, etc.), mais aussi - et surtout - en le replaçant dans une problématique de la longue durée. À lui seul, ce dernier point suffirait à valider définitivement la pertinence de cette question.

\section{Bibliographie}

J. BLACK, 1991, A Military Revolution? Military Change and European Society 1550-1800, Londres, Macmillan, coll. « Studies in European History ».

B. M. DOWNING, 1992, The Military Revolution and Political Change-Origins of Democracy in Early Modern Europe, Princeton, New Jersey, Princeton University Press.

J. CHAGNIOT, 2001, Guerre et société à l'époque moderne, Paris, PUF, coll. « Nouvelle Clio ». 
C. S. GRAY, 2002, Strategy for Chaos - Revolutions in Military Affairs and the Evidence of History, Londres, Frank Cass.

J. KLEIN et T. de MONTBRIAL (dir.), 2000, Dictionnaire de stratégie, Paris, PUF (voir, en particulier, les articles de A. Joxe, J. Sapir et L. Henninger, consacrés aux différentes « révolutions militaires »).

M. KNOX et W. MURRAY (dir.), 2001, The Dynamics of Military Revolution 13002050, Cambridge, Cambridge University Press.

G. PARKER, 1993, La révolution militaire - La guerre et l'essor de l'Occident 15001800, trad. J. Joba, Paris, Gallimard, coll. « Bibliothèque des histoires ».

C. J. ROGERS (dir.), 1995, The Military Revolution Debate-Readings on the Military Transformation of Early Modern Europe, Boulder, Colorado, Westview Press.

J. J. SCHNEIDER, 1994, The Structure of Strategic Revolution - Total War and the Roots of the Soviet Warfare State, Novato, Californie, Presidio Press. 